\title{
Extension of Jensen's inequality to affine combinations
}

\section{Zlatko Pavić}

\section{"Correspondence:}

Zlatko.Pavic@sfsb.hr

Mechanical Engineering Faculty in Slavonski Brod, University of Osijek,

Trg Ivane Brlić Mažuranić 2,

Slavonski Brod, 35000, Croatia

\begin{abstract}
The article provides the generalization of Jensen's inequality for convex functions on the line segments. The main and preliminary inequalities are expressed in discrete form using affine combinations that can be reduced to convex combinations. The resulting quasi-arithmetic means are used to extend the two well-known inequalities. MSC: $26 \mathrm{~A} 51 ; 26 \mathrm{D} 15$

Keywords: convex combination; affine combination; convex function; Jensen's inequality
\end{abstract}

\section{Introduction}

First of all, we recapitulate the basic notations of affinity and convexity relating to the binomial combinations. Let $\mathcal{X}$ be a real vector space.

A set $\mathcal{A} \subseteq \mathcal{X}$ is affine if it contains all binomial affine combinations in $\mathcal{A}$, that is, the combinations $\alpha a+\beta b$ of points $a, b \in \mathcal{A}$ and coefficients $\alpha, \beta \in \mathbb{R}$ of the sum $\alpha+\beta=1$. The affine hull of a set $\mathcal{S} \subseteq \mathcal{X}$ as the smallest affine set that contains $\mathcal{S}$ is denoted aff $\mathcal{S}$. A function $f: \mathcal{A} \rightarrow \mathbb{R}$ is affine if the equality

$$
f(\alpha a+\beta b)=\alpha f(a)+\beta f(b)
$$

holds for all binomial affine combinations $\alpha a+\beta b$ in $\mathcal{A}$.

A set $\mathcal{C} \subseteq \mathcal{X}$ is convex if it contains all binomial convex combinations in $\mathcal{C}$, that is, the combinations $\alpha a+\beta b$ of points $a, b \in \mathcal{C}$ and non-negative coefficients $\alpha, \beta \in \mathbb{R}$ of the sum $\alpha+\beta=1$. The convex hull of a set $\mathcal{S} \subseteq \mathcal{X}$ as the smallest convex set that contains $\mathcal{S}$ is denoted conv $\mathcal{S}$. A function $f: \mathcal{C} \rightarrow \mathbb{R}$ is convex if the inequality

$$
f(\alpha a+\beta b) \leq \alpha f(a)+\beta f(b)
$$

holds for all binomial convex combinations $\alpha a+\beta b$ in $\mathcal{C}$.

Using mathematical induction, it can be proved that everything said above is true for all $n$-membered affine or convex combinations. In this way, Jensen (see [1]) has extended the inequality in equation (1.2).

We will consider affine and convex combinations of the real numbers, the case where $\mathcal{X}=\mathbb{R}$. Take real numbers $a$ and $b$ such that $a \neq b$. Every number $x \in \mathbb{R}$ can be uniquely

\section{Springer}

C2014 Pavić; licensee Springer. This is an Open Access article distributed under the terms of the Creative Commons Attribution License (http://creativecommons.org/licenses/by/2.0), which permits unrestricted use, distribution, and reproduction in any medium, provided the original work is properly cited. 
presented as the binomial affine combination

$$
x=\frac{b-x}{b-a} a+\frac{x-a}{b-a} b,
$$

which is convex if, and only if, the number $x$ belongs to the interval $\operatorname{conv}\{a, b\}$. Given the function $f: \mathbb{R} \rightarrow \mathbb{R}$, let $f_{\{a, b\}}^{\text {line }}: \mathbb{R} \rightarrow \mathbb{R}$ be the function of the chord line passing through the points $A(a, f(a))$ and $B(b, f(b))$ of the graph of $f$. Applying the affinity of $f_{\{a, b\}}^{\text {line }}$ to the combination in equation (1.3), we get

$$
f_{\{a, b\}}^{\mathrm{line}}(x)=\frac{b-x}{b-a} f(a)+\frac{x-a}{b-a} f(b) .
$$

If the function $f$ is convex, then we have the inequality

$$
f(x) \leq f_{\{a, b\}}^{\text {line }}(x) \quad \text { if } x \in \operatorname{conv}\{a, b\}
$$

and the reverse inequality

$$
f(x) \geq f_{\{a, b\}}^{\text {line }}(x) \quad \text { if } x \notin \operatorname{conv}\{a, b\} \backslash\{a, b\} .
$$

A wide area of convex analysis including convex functions and their inequalities is covered in [2]. The practical applications of convex analysis are presented in [3].

A brief scientific-historic background on Jensen's inequality follows at the end of this introduction. Because of its attractiveness, Jensen's and related inequalities were studied during the whole last century. So, there are Steffensen's, Brunk's, and Olkin's inequality. In this century the research goes on, and we got Jensen-Mercer's and Mercer-Steffensen's inequality. For information as regards these inequalities, one may refer to papers [4-7] and [8].

\section{Generalization and reversal of Jensen-Mercer's inequality}

In order to generalize Jensen's inequality, we firstly consider Jensen-Mercer's inequality. A generalization of this inequality is given in Corollary 2.2, and the reverse inequality is specified in Corollary 2.4. The presentation of the section is based on the affine combinations in equation (2.1), and the simple inequality in equation (2.2).

Let $\alpha, \beta, \gamma \in \mathbb{R}$ be coefficients such that $\alpha+\beta-\gamma=1$. Let $a, b, c \in \mathbb{R}$ be points. We consider the affine combination $\alpha a+\beta b-\gamma c$. Including the affine combination $c=\lambda a+\mu b$ assuming that $\lambda+\mu=1$, we get the binomial form

$$
\alpha a+\beta b-\gamma c=(\alpha-\gamma \lambda) a+(\beta-\gamma \mu) b
$$

Lemma 2.1 Let $\alpha, \beta, \gamma \in[0,1]$ be coefficients such that $\alpha+\beta-\gamma=1$. Let $a, b, c \in \mathbb{R}$ be points such that $c \in \operatorname{conv}\{a, b\}$.

Then the affine combination $\alpha a+\beta b-\gamma c$ is in $\operatorname{conv}\{a, b\}$, and every convex function $f: \operatorname{conv}\{a, b\} \rightarrow \mathbb{R}$ satisfies the inequality

$$
f(\alpha a+\beta b-\gamma c) \leq \alpha f(a)+\beta f(b)-\gamma f(c) .
$$


Proof The condition $c=\lambda a+\mu b \in \operatorname{conv}\{a, b\}$ involves $\lambda, \mu \in[0,1]$. Then the binomial combination of the right-hand side in (2.1) is convex since its coefficients $\alpha-\gamma \lambda \geq \alpha-\gamma=$ $1-\beta \geq 0$ and also $\beta-\gamma \mu \geq 0$. So, the combination $\alpha a+\beta b-\gamma c$ belongs to $\operatorname{conv}\{a, b\}$.

Since the inequality in equation (2.2) is trivially true for $a=b$, we suppose that $a \neq b$. Applying the inequality in equation (1.5), and the affinity of $f_{\{a, b\}}^{\text {line }}$, we get

$$
\begin{aligned}
f(\alpha a+\beta b-\gamma c) & \leq f_{\{a, b\}}^{\text {line }}(\alpha a+\beta b-\gamma c) \\
& =\alpha f(a)+\beta f(b)-\gamma f_{\{a, b\}}^{\text {line }}(c) \\
& \leq \alpha f(a)+\beta f(b)-\gamma f(c)
\end{aligned}
$$

because $f_{\{a, b\}}^{\text {line }}(c) \geq f(c)$.

The inequality in equation (2.2) can also be proved applying the convexity of the function $f$ to the right-hand side of the representation in equation (2.1), wherein we use the inequality $f(c) \leq \lambda f(a)+\mu f(b)$.

Corollary 2.2 Let $\alpha, \beta, \gamma \in[0,1]$ and $\gamma_{i} \in[0,1]$ be coefficients such that $\alpha+\beta-\gamma=$ $\sum_{i=1}^{n} \gamma_{i}=1$. Let $a, b, c_{i} \in \mathbb{R}$ be points such that all $c_{i} \in \operatorname{conv}\{a, b\}$.

Then the affine combination $\alpha a+\beta b-\gamma \sum_{i=1}^{n} \gamma_{i} c_{i}$ is in $\operatorname{conv}\{a, b\}$, and every convex function $f: \operatorname{conv}\{a, b\} \rightarrow \mathbb{R}$ satisfies the inequality

$$
f\left(\alpha a+\beta b-\gamma \sum_{i=1}^{n} \gamma_{i} c_{i}\right) \leq \alpha f(a)+\beta f(b)-\gamma \sum_{i=1}^{n} \gamma_{i} f\left(c_{i}\right) .
$$

As demonstrated above, to prove the inequality in equation (2.6) it is not necessary to use the Jensen inequality. Lemma 2.1 and Corollary 2.2 are also valid for $\gamma \in[-1,1]$ because then the observed affine combinations with $\gamma \leq 0$ become convex, and the associated inequalities follow from Jensen's inequality. The combinations including $\gamma \in[-1,1]$ were observed in [9, Corollary 11 and Theorem 12] additionally using a monotone function $g$.

If $\alpha=\beta=\gamma=1$, then the inequality in equation (2.6) is reduced to Mercer's variant of Jensen's inequality obtained in [6]. Another generalization of Mercer's result was achieved in [10] using the majorization assumptions. The importance and impact of the majorization theory to inequalities can be found in [11].

Lemma 2.3 Let $\alpha, \beta, \gamma \in[1, \infty)$ be coefficients such that $\alpha+\beta-\gamma=1$. Let $a, b, c \in \mathbb{R}$ be points such that $c \notin \operatorname{conv}\{a, b\} \backslash\{a, b\}$.

Then the affine combination $\alpha a+\beta b-\gamma c$ is not in $\operatorname{conv}\{a, b\} \backslash\{a, b\}$, and every convex function $f: \operatorname{conv}\{a, b, c\} \rightarrow \mathbb{R}$ satisfies the inequality

$$
f(\alpha a+\beta b-\gamma c) \geq \alpha f(a)+\beta f(b)-\gamma f(c) .
$$

Proof The condition $c=\lambda a+\mu b \notin \operatorname{conv}\{a, b\} \backslash\{a, b\}$ entails $\lambda \leq 0$ or $\lambda \geq 1$, and the first coefficient of the binomial form of (2.1) satisfies $\alpha-\gamma \lambda \geq \alpha \geq 1$ if $\lambda \leq 0$, or $\alpha-\gamma \lambda \leq \alpha-\gamma=$ $1-\beta \leq 0$ if $\lambda \geq 1$. So, the combination $\alpha a+\beta b-\gamma$ does not belong to $\operatorname{conv}\{a, b\} \backslash\{a, b\}$. Applying the inequality in (1.6), we get the series of inequalities as in equations (2.3), (2.4), and (2.5), but with the reverse inequality signs. 
It is not necessary to require $\gamma \in[1, \infty)$ in Lemma 2.3 , it follows from the conditions $\alpha, \beta \in[1, \infty)$ and $\alpha+\beta-\gamma=1$.

Corollary 2.4 Let $\alpha, \beta, \gamma \in[1, \infty)$ and $\gamma_{i} \in[0,1]$ be coefficients such that $\alpha+\beta-\gamma=$ $\sum_{i=1}^{n} \gamma_{i}=1$. Let $a, b, c_{i} \in \mathbb{R}$ be points such that any $c_{i} \notin \operatorname{conv}\{a, b\} \backslash\{a, b\}$ and the convex combination $\sum_{i=1}^{n} \gamma_{i} c_{i} \notin \operatorname{conv}\{a, b\} \backslash\{a, b\}$.

Then the affine combination $\alpha a+\beta b-\gamma \sum_{i=1}^{n} \gamma_{i} c_{i}$ is not in $\operatorname{conv}\{a, b\} \backslash\{a, b\}$, and every convex function $f: \operatorname{conv}\left\{a, b, c_{i}\right\} \rightarrow \mathbb{R}$ satisfies the inequality

$$
f\left(\alpha a+\beta b-\gamma \sum_{i=1}^{n} \gamma_{i} c_{i}\right) \geq \alpha f(a)+\beta f(b)-\gamma \sum_{i=1}^{n} \gamma_{i} f\left(c_{i}\right) .
$$

\section{Main results}

The aim of the paper is to generalize the inequality in equation (2.2). The first step is already done in Corollary 2.2 in the expression of the inequality in equation (2.6), presenting the point $c$ as the convex combination. We are going to proceed in the same way with the points $a$ and $b$. The main theorem follows.

Theorem 3.1 Let $\alpha_{i}, \beta_{j}, \gamma_{k} \geq 0$ be coefficients with the sums $\alpha=\sum_{i=1}^{n} \alpha_{i}, \beta=\sum_{j=1}^{m} \beta_{j}$, and $\gamma=\sum_{k=1}^{l} \gamma_{k}$ satisfying $0<\alpha, \beta \leq 1$ and $\alpha+\beta-\gamma=1$. Let $a_{i}, b_{j}, c_{k} \in \mathbb{R}$ be points such that all $c_{k} \in \operatorname{conv}\{a, b\}$ where

$$
a=\frac{1}{\alpha} \sum_{i=1}^{n} \alpha_{i} a_{i}, \quad b=\frac{1}{\beta} \sum_{j=1}^{m} \beta_{j} b_{j} .
$$

Then the affine combination

$$
\sum_{i=1}^{n} \alpha_{i} a_{i}+\sum_{j=1}^{m} \beta_{j} b_{j}-\sum_{k=1}^{l} \gamma_{k} c_{k}
$$

belongs to $\operatorname{conv}\{a, b\}$, and every convex function $f: \operatorname{conv}\left\{a_{i}, b_{j}\right\} \rightarrow \mathbb{R}$ satisfies the inequality

$$
\begin{aligned}
& f\left(\sum_{i=1}^{n} \alpha_{i} a_{i}+\sum_{j=1}^{m} \beta_{j} b_{j}-\sum_{k=1}^{l} \gamma_{k} c_{k}\right) \\
& \quad \leq \sum_{i=1}^{n} \alpha_{i} f\left(a_{i}\right)+\sum_{j=1}^{m} \beta_{j} f\left(b_{j}\right)-\sum_{k=1}^{l} \gamma_{k} f\left(c_{k}\right) .
\end{aligned}
$$

Iff is concave, then the reverse inequality is valid in equation (3.3). Iff is affine, then the equality is valid in equation (3.3).

Proof Note that $\gamma \leq \alpha, \beta$. Prove the theorem for the convex function $f$.

If $\gamma=0$, the combination in equation (3.2) takes the convex form $\alpha a+\beta b$ belonging to $\operatorname{conv}\{a, b\}$, and the inequality in equation (3.3) is reduced to Jensen's inequality.

If $\gamma>0$, then, including points $a, b$, and

$$
c=\frac{1}{\gamma} \sum_{k=1}^{l} \gamma_{k} c_{k}
$$


in equation (3.2), we get the combination $\alpha a+\beta b-\gamma c$ which belongs to $\operatorname{conv}\{a, b\}$ by Lemma 2.1. The inequality in equation (3.3) is trivially true for $a=b$. So, we assume that $a \neq b$ and use the function $f_{\{a, b\}}^{\text {line }}$. Applying the affinity of $f_{\{a, b\}}^{\text {line }}$ to the convex combination in equation (3.4), and respecting the inequalities $f_{\{a, b\}}^{\text {line }}\left(c_{k}\right) \geq f\left(c_{k}\right)$, we have

$$
f_{\{a, b\}}^{\text {line }}(c)=\frac{1}{\gamma} \sum_{k=1}^{l} \gamma_{k} f_{\{a, b\}}^{\text {line }}\left(c_{k}\right) \geq \frac{1}{\gamma} \sum_{k=1}^{l} \gamma_{k} f\left(c_{k}\right)
$$

Using the inequality in equation (2.4), applying Jensen's inequality to $f(a)$ and $f(b)$, and finally using the inequality in equation (3.5) respecting the minus, we get

$$
\begin{aligned}
f\left(\sum_{i=1}^{n} \alpha_{i} a_{i}+\sum_{j=1}^{m} \beta_{j} b_{j}-\sum_{k=1}^{l} \gamma_{k} c_{k}\right) & \leq \alpha f(a)+\beta f(b)-\gamma f_{\{a, b\}}^{\text {line }}(c) \\
& \leq \sum_{i=1}^{n} \alpha_{i} f\left(a_{i}\right)+\sum_{j=1}^{m} \beta_{j} f\left(b_{j}\right)-\sum_{k=1}^{l} \gamma_{k} f\left(c_{k}\right)
\end{aligned}
$$

completing the proof.

Corollary 3.2 Let all the assumptions of Theorem 3.1 be fully satisfied. Let $\sum_{r=1}^{s} \lambda_{r} x_{r}, x_{r} \in$ $\operatorname{conv}\{a, b\}$, and $\lambda a+\mu b$ be convex combinations having the common center with the affine combination in equation (3.2), that is,

$$
\sum_{r=1}^{s} \lambda_{r} x_{r}=\lambda a+\mu b=\sum_{i=1}^{n} \alpha_{i} a_{i}+\sum_{j=1}^{m} \beta_{j} b_{j}-\sum_{k=1}^{l} \gamma_{k} c_{k}
$$

Then every convex function $f: \operatorname{conv}\left\{a_{i}, b_{j}\right\} \rightarrow \mathbb{R}$ satisfies the double inequality

$$
\sum_{r=1}^{s} \lambda_{r} f\left(x_{r}\right) \leq \lambda f(a)+\mu f(b) \leq \sum_{i=1}^{n} \alpha_{i} f\left(a_{i}\right)+\sum_{j=1}^{m} \beta_{j} f\left(b_{j}\right)-\sum_{k=1}^{l} \gamma_{k} f\left(c_{k}\right) .
$$

Iff is concave, then the reverse inequalities are valid in equation (3.7). Iff is affine, then the equalities are valid in equation (3.7).

Proof Relying on the function $f_{\{a, b\}}^{\text {line }}$ and the Jensen inequality, we derive the series of inequalities

$$
\begin{aligned}
\sum_{r=1}^{s} \lambda_{r} f\left(x_{r}\right) & \leq \sum_{r=1}^{s} \lambda_{r} f_{\{a, b\}}^{\text {line }}\left(x_{r}\right)=f_{\{a, b\}}^{\text {line }}\left(\sum_{r=1}^{s} \lambda_{r} x_{r}\right)=f_{\{a, b\}}^{\text {line }}(\lambda a+\mu b)=\lambda f(a)+\mu f(b) \\
& =f_{\{a, b\}}^{\text {line }}\left(\alpha a+\beta b-\sum_{k=1}^{l} \gamma_{k} c_{k}\right)=\alpha f(a)+\beta f(b)-\sum_{k=1}^{l} \gamma_{k} f_{\{a, b\}}^{\text {line }}\left(c_{k}\right) \\
& \leq \sum_{i=1}^{n} \alpha_{i} f\left(a_{i}\right)+\sum_{j=1}^{m} \beta_{j} f\left(b_{j}\right)-\sum_{k=1}^{l} \gamma_{k} f\left(c_{k}\right)
\end{aligned}
$$

containing the double inequality in equation (3.7). 
Corollary 3.3 Let all the assumptions of Theorem 3.1 be fully satisfied. Let $\sum_{r=1}^{s} \lambda_{r} x_{r}$ be a convex combination with points $x_{r} \in \operatorname{conv}\{a, b\}$ such that

$$
\sum_{r=1}^{s} \lambda_{r} x_{r}+\sum_{k=1}^{l} \gamma_{k} c_{k}=\sum_{i=1}^{n} \alpha_{i} a_{i}+\sum_{j=1}^{m} \beta_{j} b_{j} .
$$

Then every convex function $f: \operatorname{conv}\left\{a_{i}, b_{j}\right\} \rightarrow \mathbb{R}$ satisfies the inequality

$$
\sum_{r=1}^{s} \lambda_{r} f\left(x_{r}\right)+\sum_{k=1}^{l} \gamma_{k} f\left(c_{k}\right) \leq \sum_{i=1}^{n} \alpha_{i} f\left(a_{i}\right)+\sum_{j=1}^{m} \beta_{j} f\left(b_{j}\right)
$$

We finish the section with the basic integral variant of the main theorem. In the corollary that follows we use segments with different endpoints.

Corollary 3.4 Let $\alpha, \beta, \gamma \in[0,1]$ be coefficients such that $\alpha+\beta-\gamma=1$. Let $\left[a_{1}, a_{2}\right],\left[b_{1}, b_{2}\right]$, $\left[c_{1}, c_{2}\right] \subset \mathbb{R}$ be segments such that

$$
\left[c_{1}, c_{2}\right] \subseteq\left[\frac{a_{1}+a_{2}}{2}, \frac{b_{1}+b_{2}}{2}\right]
$$

Then the integral sum

$$
\bar{x}=\frac{\alpha}{a_{2}-a_{1}} \int_{a_{1}}^{a_{2}} x d x+\frac{\beta}{b_{2}-b_{1}} \int_{b_{1}}^{b_{2}} x d x-\frac{\gamma}{c_{2}-c_{1}} \int_{c_{1}}^{c_{2}} x d x
$$

belongs to $\left[\left(a_{1}+a_{2}\right) / 2,\left(b_{1}+b_{2}\right) / 2\right]$, and every convex function $f:\left[a_{1}, b_{2}\right] \rightarrow \mathbb{R}$ satisfies the inequality

$$
f(\bar{x}) \leq \frac{\alpha}{a_{2}-a_{1}} \int_{a_{1}}^{a_{2}} f(x) d x+\frac{\beta}{b_{2}-b_{1}} \int_{b_{1}}^{b_{2}} f(x) d x-\frac{\gamma}{c_{2}-c_{1}} \int_{c_{1}}^{c_{2}} f(x) d x .
$$

Iff is concave, then the reverse inequality is valid in equation (3.11). Iff is affine, then the equality is valid in equation (3.11).

Proof The sum of (3.10) is equal to the affine combination

$$
\bar{x}=\alpha \frac{a_{1}+a_{2}}{2}+\beta \frac{b_{1}+b_{1}}{2}-\gamma \frac{c_{1}+c_{2}}{2}
$$

belonging to $\left[\left(a_{1}+a_{2}\right) / 2,\left(b_{1}+b_{2}\right) / 2\right]$ by Lemma 2.1. Given the positive integer $n$, we can construct the affine combination

$$
\bar{x}_{n}=\sum_{i=1}^{n} \alpha_{n i} a_{n i}+\sum_{j=1}^{n} \beta_{n j} b_{n j}-\sum_{k=1}^{n} \gamma_{n k} c_{n k}
$$

which approaches the integral sum in equation (3.10) as $n$ approaches infinity. Let us show how it looks in the example of the first member of the above combination.

We make the partition $\left[a_{1}, a_{2}\right]=\bigcup_{i=1}^{n}\left[\left(a_{1}\right)_{n i},\left(a_{2}\right)_{n i}\right]$ where all subsegments have the same length $\left(a_{2}-a_{1}\right) / n$, and the adjacent subsegments have a common endpoint. If we take 
points $a_{n i} \in\left[\left(a_{1}\right)_{n i},\left(a_{2}\right)_{n i}\right]$, then we have

$$
\lim _{n \rightarrow \infty} \sum_{i=1}^{n} \frac{1}{n} a_{n i}=\lim _{n \rightarrow \infty} \sum_{i=1}^{n} \frac{\left(a_{2}\right)_{n i}-\left(a_{1}\right)_{n i}}{a_{2}-a_{1}} a_{n i}=\frac{1}{a_{2}-a_{1}} \int_{a_{1}}^{a_{2}} x d x
$$

suggesting to take $\alpha_{n i}=\alpha / n$.

Applying Theorem 3.1 to the affine combination in equation (3.13) and the convex function $f$, we get

$$
f\left(\bar{x}_{n}\right) \leq \sum_{i=1}^{n} \alpha_{n i} f\left(a_{n i}\right)+\sum_{j=1}^{n} \beta_{n j} f\left(b_{n j}\right)-\sum_{k=1}^{n} \gamma_{n k} f\left(c_{n k}\right),
$$

and letting $n$ to infinity, we obtain the integral inequality in equation (3.11).

\section{Application to quasi-arithmetic means}

Let $\mathcal{I} \subseteq \mathbb{R}$ be an interval. Applying the Jensen inequality to the means, we use strictly monotone continuous functions $\varphi, \psi: \mathcal{I} \rightarrow \mathbb{R}$ such that the function $\psi \circ \varphi^{-1}: \varphi(\mathcal{I}) \rightarrow \mathbb{R}$ is convex. In this case, we say that $\psi$ is convex with respect to $\varphi$, or $\psi$ is $\varphi$-convex. The same notation is used for the concavity. If the function $\psi$ is $\varphi$-convex and $\varphi$-concave, we can say that it is $\varphi$-affine.

Suppose that

$$
\bar{x}=\sum_{i=1}^{n} \alpha_{i} a_{i}+\sum_{j=1}^{m} \beta_{j} b_{j}-\sum_{k=1}^{l} \gamma_{k} c_{k}
$$

is an affine combination as in Theorem 3.1, and $\varphi: \mathcal{I} \rightarrow \mathbb{R}$ is a strictly monotone continuous function where $\mathcal{I}=\operatorname{conv}\left\{a_{i}, b_{j}\right\}$. The discrete $\varphi$-quasi-arithmetic mean of the combination $\bar{x}$ can be defined as the point

$$
M_{\varphi}(\bar{x})=\varphi^{-1}\left(\sum_{i=1}^{n} \alpha_{i} \varphi\left(a_{i}\right)+\sum_{j=1}^{m} \beta_{j} \varphi\left(b_{j}\right)-\sum_{k=1}^{l} \gamma_{k} \varphi\left(c_{k}\right)\right)
$$

belonging to $\operatorname{conv}\{a, b\}$, because the affine combination enclosed in parentheses is located in $\varphi(\operatorname{conv}\{a, b\})$. The quasi-arithmetic means defined in equation (4.2) are invariant with respect to the affinity, that is, the equality

$$
M_{\varphi}(\bar{x})=M_{u \varphi+v}(\bar{x})
$$

holds for all pairs of real numbers $u \neq 0$ and $v$.

The order of the pair of quasi-arithmetic means $M_{\varphi}$ and $M_{\psi}$ depends on convexity of the function $\psi \circ \varphi^{-1}$ and monotonicity of the function $\psi$, as follows.

Corollary 4.1 Let $\bar{x}$ be an affine combination as in equation (4.1) satisfying all the assumptions of Theorem 3.1. Let $\varphi, \psi: \mathcal{I} \rightarrow \mathbb{R}$ be strictly monotone continuous functions where $\mathcal{I}=\operatorname{conv}\left\{a_{i}, b_{j}\right\}$.

If $\psi$ is either $\varphi$-convex and increasing or $\varphi$-concave and decreasing, then we have the inequality

$$
M_{\varphi}(\bar{x}) \leq M_{\psi}(\bar{x})
$$


If $\psi$ is either $\varphi$-convex and decreasing or $\varphi$-concave and increasing, then we have the reverse inequality in equation (4.4).

If $\psi$ is $\varphi$-affine, then the equality is valid in equation (4.4).

Proof We prove the case that the function $\psi$ is $\varphi$-convex and increasing. Put the set $\mathcal{J}=$ $\varphi(\mathcal{I})=\operatorname{conv}\left\{\varphi\left(a_{i}\right), \varphi\left(b_{j}\right)\right\}$. Applying the inequality in equation (3.3) of Theorem 3.1 to the affine combination

$$
\bar{x}_{\varphi}=\sum_{i=1}^{n} \alpha_{i} \varphi\left(a_{i}\right)+\sum_{j=1}^{m} \beta_{j} \varphi\left(b_{j}\right)-\sum_{k=1}^{l} \gamma_{k} \varphi\left(c_{k}\right),
$$

which is in the set $\varphi(\operatorname{conv}\{a, b\})$, and the convex function $f=\psi \circ \varphi^{-1}: \mathcal{J} \rightarrow \mathbb{R}$, we get

$$
\psi \circ \varphi^{-1}\left(\bar{x}_{\varphi}\right) \leq \bar{x}_{\psi}
$$

Assigning the increasing function $\psi^{-1}$ to the above inequality, we attain

$$
M_{\varphi}(\bar{x})=\varphi^{-1}\left(\bar{x}_{\varphi}\right) \leq \psi^{-1}\left(\bar{x}_{\psi}\right)=M_{\psi}(\bar{x}),
$$

which finishes the proof.

The inequality in equation (4.4) may further be applied to the power means. The monotonicity of these power means is also valid. We will only derive the formula of the harmonic-geometric-arithmetic mean inequality.

Corollary 4.2 If $\bar{x}$ is an affine combination as in equation (4.1) satisfying all the assumptions of Theorem 3.1 with the addition that all $a_{i}, b_{j}>0$, then we have the harmonicgeometric-arithmetic mean inequality

$$
\begin{aligned}
\left(\sum_{i=1}^{n} \frac{\alpha_{i}}{a_{i}}+\sum_{j=1}^{m} \frac{\beta_{j}}{b_{j}}-\sum_{k=1}^{l} \frac{\gamma_{k}}{c_{k}}\right)^{-1} & \leq \prod_{i=1}^{n} a_{i}^{\alpha_{i}} \prod_{j=1}^{m} b_{j}^{\beta_{j}} \prod_{k=1}^{l} c_{k}^{-\gamma_{k}} \\
& \leq \sum_{i=1}^{n} \alpha_{i} a_{i}+\sum_{j=1}^{m} \beta_{j} b_{j}-\sum_{k=1}^{l} \gamma_{k} c_{k} .
\end{aligned}
$$

Proof Let $\varphi_{-1}(x)=x^{-1}, \varphi_{0}(x)=\ln x$, and $\varphi_{1}(x)=x$. Applying the inequality in equation (4.4), first to the pair $\varphi_{-1}$ and $\varphi_{0}$, and then to the pair $\varphi_{0}$ and $\varphi_{1}$, we get $M_{-1} \leq M_{0} \leq M_{1}$ representing the inequality in equation (4.6).

The general approach to the theory of means and their inequalities was presented in [12]. Different forms of quasi-arithmetic means were considered in [13].

\section{Application to other inequalities}

The essential inequalities in equations (2.2) and (2.7) can be used to extend the Bernoulli and the Young inequality. Let us start with a simplified case of the mean inequality in equation (4.6). 
Corollary 5.1 If numbers $\alpha, \beta, \gamma \in[0,1]$ satisfy $\alpha+\beta-\gamma=1$, then the double inequality

$$
\left(\frac{\alpha}{a}+\frac{\beta}{b}-\frac{\gamma}{c}\right)^{-1} \leq \frac{a^{\alpha} b^{\beta}}{c^{\gamma}} \leq \alpha a+\beta b-\gamma c
$$

holds for all numbers $a, b, c>0$ such that $c \in \operatorname{conv}\{a, b\}$.

If $\alpha, \beta, \gamma \in[1, \infty)$ satisfy $\alpha+\beta-\gamma=1$, then the reverse double inequality is valid in equation (5.1) for all $a, b, c>0$ such that $c \notin \operatorname{conv}\{a, b\} \backslash\{a, b\}$.

Proof The inequality in equation (5.1) follows from the inequality in equation (4.6) with $\alpha_{i}=\alpha, \beta_{j}=\beta, \gamma_{k}=\gamma$ and $a_{i}=a, b_{j}=b, c_{k}=c$. So, it is actually a consequence of the inequality in equation (2.2).

The reverse inequality of equation (5.1) can be obtained from the inequality in equation (2.7) using the functions $\varphi_{-1}, \varphi_{0}$, and $\varphi_{1}$.

The previous corollary with a suitable choice of the coefficients and the points will be applied in the following two examples.

Example 5.2 If numbers $p, q, r \in[0,1]$ satisfy $p+q-r=1$, then the double inequality

$$
\left(\frac{p}{1+x}+\frac{q}{1+y}-\frac{r}{1+z}\right)^{-1} \leq \frac{(1+x)^{p}(1+y)^{q}}{(1+z)^{r}} \leq 1+p x+q y-r z
$$

holds for all numbers $x, y, z>-1$ such that $z \in \operatorname{conv}\{x, y\}$.

If $p, q, r \in[1, \infty)$ satisfy $p+q-r=1$, then the reverse double inequality is valid in equation (5.2) for all $x, y, z>-1$ such that $z \notin \operatorname{conv}\{x, y\} \backslash\{x, y\}$.

Proof Corollary 5.1 should be applied to the coefficients $\alpha=p, \beta=q, \gamma=r$ and the points $a=1+x, b=1+y, c=1+z$.

The inequality

$$
\frac{1+t}{1+t-p t} \leq(1+t)^{p} \leq 1+p t
$$

applying to $p \in[0,1]$ and $t>-1$ follows from the inequality in equation (5.2) putting first $y=z=0$ and taking $t=x \in(-1,0]$, then putting $x=z=0$ and taking $t=y \in[0, \infty)$. In the same way, from the reverse inequality of equation (5.2) we can obtain the reverse inequality of equation (5.3) respecting $p \in[1, \infty)$ and $t>-1$. The right-hand sides of these inequalities are the well-known Bernoulli's inequalities.

Example 5.3 If numbers $p, q \in[1, \infty)$ and $r \in[0,1]$ satisfy $1 / p+1 / q-r=1$, then the double inequality

$$
\left(\frac{x^{-p}}{p}+\frac{y^{-q}}{q}-r z^{-1}\right)^{-1} \leq \frac{x y}{z^{r}} \leq \frac{x^{p}}{p}+\frac{y^{q}}{q}-r z
$$

holds for all numbers $x, y, z>0$ such that $z \in \operatorname{conv}\left\{x^{p}, y^{q}\right\}$. 
If $p, q \in(0,1]$ and $r \in[1, \infty)$ satisfy $1 / p+1 / q-r=1$, then the reverse double inequality is valid in equation (5.4) for all $x, y, z>0$ such that $z \notin \operatorname{conv}\left\{x^{p}, y^{q}\right\} \backslash\left\{x^{p}, y^{q}\right\}$.

Proof Corollary 5.1 should be applied to the coefficients $\alpha=1 / p, \beta=1 / q, \gamma=r$ and the points $a=x^{p}, b=y^{q}, c=z$.

Taking $r=0$ in equation (5.4), we have the inequality

$$
\left(\frac{x^{-p}}{p}+\frac{y^{-q}}{q}\right)^{-1} \leq x y \leq \frac{x^{p}}{p}+\frac{y^{q}}{q}
$$

which applies to numbers $p, q \in(1, \infty)$ with the sum $1 / p+1 / q=1$, as well as to numbers $x, y>0$. The right-hand side of the inequality in equation (5.5) represents the discrete form of Young's inequality.

\section{Competing interests}

The author declares that they have no competing interests.

Received: 4 February 2014 Accepted: 8 July 2014 Published: 19 Aug 2014

\section{References}

1. Jensen, JLWV: Om konvekse Funktioner og Uligheder mellem Middelværdier. Nyt Tidsskr. Math. B 16, 49-68 (1905)

2. Pečarić, JE, Proschan, F, Tong, YL: Convex Functions, Partial Orderings, and Statistical Applications. Academic Press, New York (1992)

3. Rockafellar, RT: Convex Analysis. Princeton University Press, Princeton (1972)

4. Pečarić, JE: A simple proof of the Jensen-Steffensen inequality. Am. Math. Mon. 91, 195-196 (1984)

5. Bjelica, M: Refinement and converse of Brunk-Olkin inequality. J. Math. Anal. Appl. 272, $462-467$ (1998)

6. Mercer, AMcD: A variant of Jensen's inequality. J. Inequal. Pure Appl. Math. 4, Article 73 (2003)

7. Pečarić, JE, Perušić, A, Smoljak, K: Mercer and Wu-Srivastava generalisations of Steffensen's inequality. Appl. Math. Comput. 219, 10548-10558 (2013)

8. Pavić, Z: Generalizations of Jensen-Mercer's inequality. J. Pure Appl. Math. Adv. Appl. 11, 19-36 (2014)

9. Pavić, Z: The applications of functional variants of Jensen's inequality. J. Funct. Spaces Appl. 2013, Article ID 194830 (2013)

10. Niezgoda, M: A generalization of Mercer's result on convex functions. Nonlinear Anal., Theory Methods Appl. 71 , 2771-2779 (2009)

11. Marshall, AW, Olkin, I: Inequalities: Theory of Majorization and Its Applications. Academic Press, New York (1979)

12. Bullen, PS, Mitrinović, DS, Vasić, PM: Means and Their Inequalities. Reidel, Dordrecht (1988)

13. Mićić, J, Pavić, Z, Pečarić, J: The inequalities for quasiarithmetic means. Abstr. Appl. Anal. 2012, Article ID 203145 (2012)

10.1186/1029-242X-2014-298

Cite this article as: Pavić: Extension of Jensen's inequality to affine combinations. Journal of Inequalities and Applications 2014, 2014:298

\section{Submit your manuscript to a SpringerOpen ${ }^{\circ}$ journal and benefit from:}

- Convenient online submission

- Rigorous peer review

- Immediate publication on acceptance

- Open access: articles freely available online

- High visibility within the field

- Retaining the copyright to your article

Submit your next manuscript at $>$ springeropen.com 